\title{
Elaboración De Una Tisana A Partir De Hojas De Aguacate (Persea Americana Mill), Orégano (Origanum Vulgare L.) Y Fibra De Coco
}

\author{
Brito Hanníbal \\ Palmay Paúl \\ Grupo de Investigación Ambiental y Desarrollo de la ESPOCH \\ Silva Ángel \\ Caluña Edmundo \\ Ramos Iván \\ Colaborador Facultad de Ciencias \\ Mariño Margoth \\ Romero Eliana \\ Colaborador Escuela de Ingeniería Química \\ Guadalupe Luis \\ Colaborador Centro de Idiomas ESPOCH \\ Rodríguez, Valeria
}

Grupo de Investigación en Tecnología y Atención Farmacéutica de la

ESPOCH

doi: 10.19044/esj.2016.v12n36p70 URL:http://dx.doi.org/10.19044/esj.2016.v12n36p70

\begin{abstract}
A tisane was made from the leaves of avocado, oregano and coconut fiber; with the purpose of establishing a process for obtaining this product. This research begins with the identification of the species of the vegetal elements and their respective active principles. The production process was developed at the laboratory level, it involved a set of stages of transformation of the raw material to reach the final product, considering specific variables or parameters such as: $\mathrm{PH}$, temperature, average diameter in mass, moisture of the vegetal elements and time required in certain stages. In addition, the control of conservation of active principles were carried out by comparing spectra of reception samples in grinding and subsequent sieving. The formulation of the tisane was established by considering the content of active ingredients of therapeutic interest and conducting tastings with the respective surveys, establishing the following composition per gram of tisane: $50 \%$ oregano leaves, $40 \%$ coconut fiber and $10 \%$ avocado leaves; with an acceptability of the product of $65.12 \%$ by the consumers of both genders. At
\end{abstract}


the end of the investigation, it was determined that the preparation process for the tisane includes the following steps: reception, selection, cleaning / washing, disinfection, pre-drying, dehydration, manual cutting, Prepackaging, sterilization, formulation, labeled and packaging.

Keywords: Oregano leaves Origanum vulgare L./ /Avocado leaves Persea Americana Mill//Coconut Fiber//Herbs//Active Principles/ /Manufacturing Process.

\section{Resumen}

Se procedió a la elaboración de una tisana a partir de las hojas de aguacate, orégano y fibra de coco; con la finalidad de establecer un proceso para la obtención de éste producto. Esta investigación inicia con la identificación de las especies de los elementos vegetales y sus respectivos principios activos. El procedimiento de producción se desarrolló a nivel de laboratorio, éste implicó un conjunto de etapas de transformación de la materia prima hasta llegar al producto final, considerando variables o parámetros específicos como: $\mathrm{pH}$, temperatura, diámetro promedio en masa, humedad de los elementos vegetales y tiempo requerido en determinadas etapas. Además se llevó a cabo el control de conservación de principios activos mediante la comparación de espectros de muestras de recepción en la molienda y posterior tamizado. La formulación de la tisana se estableció mediante la consideración del contenido de principios activos de interés terapéutico y la realización de degustaciones con las respectivas encuestas, estableciéndose como resultados la siguiente composición por gramo de tisana: 50\% hojas de orégano, $40 \%$ fibra de coco y $10 \%$ hojas de aguacate; con una aceptabilidad del producto de $65,12 \%$ por parte de los consumidores de ambos géneros. A la finalización de la investigación se llegó a determinar que el procedimiento de elaboración para la tisana comprende las siguientes etapas: recepción, selección, limpieza/lavado, desinfección, pre-secado, deshidratado, cortado/triturado manual, pesado, molienda-tamizado, envasado preliminar, esterilización, formulación, sacheteado y empaquetado.

Palabras Claves: Hojas de Orégano Origanum vulgare L./ /Hojas de Aguacate Persea americana Mill//Fibra de coco//Tisana//Principios Activos/ /Procedimiento de Elaboración.

\section{Introducción}

Desde la antigüedad el hombre ha utilizado los beneficios medicinales de las plantas para tratar diversas enfermedades, sin embargo con el progreso de la Química y el surgimiento de la Farmacología se opacó su utilización, no obstante siempre se ha cuestionado el hecho de que ningún químico se halla 
exento de efectos secundarios, por tal motivo la Fitomedicina sigue manteniendo vigencia en el mercado, por su capacidad para regular procesos vitales, prevenir enfermedades sin causar efecto de rebote o resistencia y ser una valiosa fuente de salud al usarlas racionalmente.

Actualmente, las plantas medicinales tienen un importante valor en la industria, principalmente en el área alimenticia, farmacéutica, gastronómica y cosmetológica. En el Ecuador industrias como: Aromas de Tungurahua, Especierías ILE C.A y Jambi Kiwa, se han destacado por su oferta de productos, tisanas a partir de plantas aromáticas (Marcillo \& Naranjo, 2012).

Hay materias primas vegetales que aún no han sido aprovechadas ni explotadas como es el caso de las hojas de aguacate y orégano, así como la fibra de coco, mismas que presentan innumerables propiedades medicinales, las cuales justifican su utilización para la producción de tisanas, cuyo producto presenta facilidad de manipulación y accesibilidad en el mercado (Valdez, 2011; Torrenegra, 2014; Cajas, 2011).

Por tanto, el objetivo de este artículo es plantear un proceso adecuado para la producción de tisanas a partir de hojas de aguacate, orégano y fibra de coco, conservando todos sus principios activos durante el proceso.

Las hojas de aguacate de la especie Persea americana Mill presenta significativas concentraciones de fósforo, potasio, calcio y magnesio. Según estudios analíticos se identificaron 28 compuestos químicos en la fracción volátil de las hojas del Persea americana Mill var. drymifolia, entre los que se menciona: diez monoterpenoides (1S- $\alpha$-pineno, L- $\beta$-pineno, sabineno, $\beta$ pineno, $\alpha$-felandreno, $\mathrm{p}$-cimeno, $1 \mathrm{R}$ - $\alpha$-pineno, eucaliptol, cis- $\beta$-terpineol y $\beta$ linalol); siete sesquiterpenoides ( $\beta$-cubebeno, $\alpha$-humuleno, cariofileno, óxido de cariofileno, germacreno D, nerolidol y Germacreno D-4-ol); seis fenilpropanoides (eugenol, eugenolmetil-éter, estragol, anetol, chavicol y chavicol-metiléter); un acetato (fenol-4-(2-propenil)-acetato) y cuatro alcanos (5-metil-tridecano, octadecano, hexadecano y heptadecano). Entre sus componentes mayoritarios se encuentran: estragol con un $38 \%$, cariofileno con un $11 \%$ y p-cimeno con un $6,8 \%$. La utilización y aprovechamiento de las propiedades medicinales del aguacate es conocida desde la antigüedad en países como México, reportándose que tribus como Totonacos, Alcozauca, Tepehua y los Nahuas utilizan la infusión de sus hojas para tratar los cólicos, dolores de estómago y de cabeza, diarrea, vómito y tos. Los chinantecos de la Asierra Madre Occidental del distrito Chinantla (Oaxaca) empleaban sus hojas como contraceptivo y emanogogo. El banco de datos del IMEPLAN enlista las siguientes propiedades terapéuticas populares de la especie Persea americana Mill var. Drymifolia como: antidiarréico, antineurálgico, vermífugo, antitumoral, antirreumático, cicatrizante, emanogogo, hemostático, expectorante, carminativo, febrífugo, estomáquico y antiinflamatorio (Valdez, 2011). Hay que destacar que su propiedad de 
emanogogo se le atribuye a la presencia del compuesto estragol, el cual actúa contra la dismenorrea a través de su actividad inhibitoria sobre la monoamina oxidasa.

Las hojas de orégano de la especie Origanum vulgare L. presentan en su composición química: $65,63 \%$ de carvacrol, $13,17 \%$ de timol, 9,81\% de $\alpha$ terpineno, 7,49\% de cariofileno, $1,39 \%$ de $\beta$-pineno y $1,86 \%$ de terpinen-4-ol (Torrenegra, 2014). Según estudios experimentales se ha revelado que las hojas del orégano presentan una potente actividad antimicrobiana, que se le atribuye a componentes como timol, carvacrol, cimeno y terpineno, los cuales actúan sobre bacterias, hongos, levaduras y virus obligándolos a cambiar su modo de acción en el organismo, inhibiendo su producción de toxinas, desintegrando su membrana celular y conduciéndolos a la muerte. La actividad antimicrobiana de los principios activos del orégano actúan específicamente sobre microorganismos como: Escherichia coli, Staphilococus aureas, Salmonella typhimurium, Candida albicans, Klebsiella pneumoniae, Enterococcus faecalis y Pseudomonas aeruginosa. La eficiencia de esta actividad se acrecienta cuando estos compuestos actúan juntos, es decir mediante el uso de la planta ya que en ésta todos los compuestos se encuentran estables y equilibrados. Su propiedad antimicrobiana lo ha hecho destacarse como un buen conservante de alimentos principalmente utilizado en quesos, productos de panadería, carnes, pescado, mariscos, frutas y verduras, ya que presenta una toxicidad nula, contribuye con las características organolépticas de sabor y olor de los alimentos permitiendo consumir productos saludables y libres de aditivos sintéticos. El contenido nutricional del orégano indica concentraciones significativas de proteínas, carbohidratos, calcio, fósforo, hierro, vitaminas B1, B2, B5, A y ácido ascórbico (Sánchez, 2013). Según la norma NTE INEN 2392:2007 (Hierbas Aromáticas. Requisitos) específica que para ésta especie su porcentaje mínimo en aceites esenciales es de 0,5\% para ser utilizado como hierba aromática. El Origanum vulgare L. posee innumerables propiedades terapéuticas que se le atribuyen a cada uno de sus principios activos, entre estas se encuentran: antimicrobiano, antioxidante, expectorante, béquico, antitusígeno, tónico, amargo excitante, emenagogo, diurético, diaforético, estrogénico, antiparasitario, antiespasmódico, dermatigénico, antiácido, antidiarreico, antimicótico, antiviral, resolutivo, estomáquico, antiacné, antiséptico, antiinflamatorio, antirreumático, cicatrizante, antimutagénico, estimulante, sedante, bactericida y carminativo. Es comúnmente utilizado en infusiones, tisanas, cataplasmas y medicamentos naturales, para tratar trastornos digestivos como flatulencia, estreñimiento, espasmos y cólicos, estimulando las funciones gástricas y biliares, además controla los niveles de ácido úrico y colesterol en el organismo, evita los cólicos menstruales y previene el cáncer de colon (Torrenegra, 2014). 
La fibra de coco de la especie Cocos nucifera de la variedad Criolla (Alta) contiene en su composición química: 42,3\% de lignina, 32,3\% de celulosa, $14,7 \%$ de pentanosa, $5,1 \%$ de grasa saponificable y $1,2 \%$ de proteínas. (Cajas, 2011) Además de concentraciones de taninos, magnesio, ácido linolénico y linoléico. Es así que el conjunto de principios activos le brinda excelentes propiedades medicinales como: antimicrobiano, antidiarreico y antioxidante; por lo que puede ser utilizado para tratar malestares digestivos como empacho, acidez estomacal, diarrea y estreñimiento reduciendo el tiempo de eliminación de forma que los carcinógenos potenciales en los alimentos dejan el colon antes.

\section{Metodología}

El proceso de elaboración de tisanas a pequeña escala se llevó a cabo en el laboratorio de Procesos Industriales de la Facultad de Ciencias ESPOCH, considerando las siguientes etapas:

Recepción: El abastecimiento de las hojas de aguacate, orégano y fibra de coco para la elaboración de tisanas es la primera etapa de la línea de producción, en la cual existe la participación del operario, el cual debe cumplir con el protocolo de equipos de protección individual como en todas las etapas siguientes.

Selección: La etapa de selección implica una observación directa de la materia prima por parte del operario, para la respectiva separación de posibles residuos sólidos existentes que no cumplan con los requerimientos de la materia prima como hojas maltratadas, tallos y fibra descompuesta.

Limpieza/ Lavado: El término limpieza hace referencia a las hojas de aguacate debido a que las impurezas (esporas) presentes en dichas hojas no son retiradas eficazmente con un lavado simple; la acción es realizada por el operario de forma manual mediante la utilización de papel absorbente, modalidad justificada por la escala baja de producción, sin embargo a nivel industrial es importante considerar una técnica específica para disminuir el tiempo requerido en esta etapa. El término lavado hace referencia a las hojas de orégano y fibra de coco, la acción es realizada por el operario mediante la utilización de agua potable.

Desinfección: La desinfección de cada material vegetal se realiza mediante la utilización de: Dióxido de cloro para alimentos $\left(\mathrm{ClO}_{2}\right)$ al $16 \%$ y $\mathrm{H}_{2} \mathrm{O}$, en una proporción $1 \mathrm{~mL}: 1 \mathrm{~L}$ respectivamente. El tiempo de desinfección es de $45 \mathrm{~min}$, con una verificación de $\mathrm{pH}$ (con tendencia a la neutralidad) al finalizar la desinfección.

Presecado: El presecado se realiza de forma natural en un intervalo de tiempo de $24 \mathrm{~h}$ a temperatura ambiente utilizado con la finalidad de reducir el consumo energético y tiempo de residencia en la etapa de deshidratación ya que el material vegetal al pasar por las etapas anteriores acumula una mayor 
humedad. Es conveniente disponer el material vegetal en capas delgadas sobre catres que se exponen al aire libre durante $24 \mathrm{~h}$, teniendo la precaución de removerlos frecuentemente y de cubrirlos o guardarlos bajo techo durante la noche para evitar que el rocío ennegrezca el producto.

Deshidratación: La etapa deshidratación se realiza en un secador tipo armario a $30{ }^{\circ} \mathrm{C}$, temperatura a la cual se evita la pérdida de los principios activos de los materiales vegetales, específicamente de los aceites esenciales ya que presentan volatilidad a altas temperaturas. Cada material es colocado en las bandejas individualmente. Es importante respetar los tiempos de secado determinados para cada material vegetal para obtener una humedad especificada.

Desfibrado-Cortado/Triturado Manual: El término cortado hace referencia a la acción realizada con la fibra de coco y el triturado manual a las hojas de aguacate, con la finalidad de que las partículas vegetales no sobrepasen el diámetro de la regulación que se ubica en la tolva de alimentación del molino. La acción es realizada por el operario de forma manual, modalidad justificada por la escala baja de producción.

Pesado: El pesado previo a la molienda-tamizado del material vegetal es considerado para establecer las pérdidas que se puede considerar como producto fino.

Molienda - Tamizado: La molienda-tamizado de cada elemento vegetal se realiza de forma individual mediante la utilización de un molino Micronizador. Cada elemento vegetal debe cumplir con el diámetro máximo de alimentación de 1 plg $(0,00254 \mathrm{~m})$ y con valores de humedad del 7\% (bh) para las hojas y $5 \%$ (bh) para la fibra de coco, valores que fueron especificados mediante pruebas experimentales y recomendados porque facilitan la reducción de tamaño de dichas materias vegetales y evitan el estrangulamiento de la materia prima en el molino. El tamiz incorporado en la cámara de molienda del equipo es de $850 \mu \mathrm{m}$ perteneciente al número de malla 20 , indicador de la granulometría del producto final.

Envasado Preliminar: El producto de descarga del Micronizador es envasado en fundas de polietileno herméticas, que aseguren la asepsia del producto durante su almacenamiento y transporte a su posterior etapa del proceso.

Esterilización: La esterilización del material particulado se realiza en autoclave a $30{ }^{\circ} \mathrm{C}$ por un intervalo de $120 \mathrm{~min}$, esta etapa se la realiza para garantizar la inocuidad completa del producto, principalmente evitar la aparición de Mohos y Levaduras, Salmonella, Escherichia coli y aerobios mesófilos, que exige la norma INEN 2532 (Especias y Condimentos. Requisitos). 
Formulación: La formulación de la tisana establece un peso de $1 \mathrm{~g}$ por cada una de ellas lo cual corresponde al 50\% de Hojas de Orégano, 40\% Fibra de Coco y 10\% Hojas de Aguacate.

Sacheteado/Empacado: El sacheteado manual implica la participación del operario para la colocación de cada elemento vegetal en el papel filtro termosellable, posteriormente se coloca el hilo de algodón y se procede al sellado a temperatura. Finalmente a cada bolsa de tisana se le coloca el papel de cobertura y se empaca las unidades en las cajas para presentación.

\section{Técnicas Específicas}

Diámetro promedio en masa de los elementos vegetales por el método del tamizado.

Se recomienda a las personas utilizar para la realización de ésta técnica: mandil, cofia, guantes y mascarilla, para mantener la asepsia en el procedimiento y el protocolo de los EPI (Equipos de Protección Individual).

Procedimiento:

$\checkmark$ Pesar las muestras de cada material vegetal, provenientes de la alimentación y descarga de la etapa de molienda - tamizado.

$\checkmark$ Armar la serie de tamices en forma descendente de acuerdo a la abertura de malla, seleccionando aquellos de acuerdo a la muestra que se va a tamizar.

$\checkmark$ Colocar la muestra en el tamiz superior, evitando pérdidas del material durante el procedimiento y luego colocar la tapa.

$\checkmark \quad$ Iniciar el movimiento de vibración, el cual se encuentra en un intervalo de $5 \mathrm{~min}$ para las hojas y $10 \mathrm{~min}$ para la fibra.

$\checkmark$ Pesar la masa del material vegetal, retenida en cada uno de los tamices. Utilizar la escobilla para extraer toda la muestra, con la finalidad de disminuir pérdidas del material.

\section{Humedad de las Hojas de Aguacate, Orégano y Fibra de Coco.}

Se recomienda a las personas utilizar para la realización de ésta técnica: mandil, cofia, guantes y mascarilla, para mantener la asepsia en el proceso y el protocolo de los EPI (Equipos de Protección Individual).

Procedimiento:

$\checkmark$ Las muestras previamente desinfectadas y pre-secadas a temperatura ambiente por un intervalo de tiempo de 24 horas, deben ser pesadas.

$\checkmark$ Encender el secador y estabilizar la temperatura a $30^{\circ} \mathrm{C}$.

$\checkmark$ Cubrir las bandejas del secador con el papel aluminio.

$\checkmark$ Colocar las muestras vegetales en las bandejas respectivas y sobre el papel aluminio.

$\checkmark$ Introducir las bandejas en el secador e iniciar la deshidratación. 
$\checkmark$ Realizar el pesaje de cada una de las muestras en un intervalo de tiempo de 25 min, hasta que la masa de las mismas sea constante.

\section{Conservación de Principios Activos. (Laboratorio de Análisis Instrumental)}

Previamente a la formulación se realiza un control en cuanto a la conservación de principios activos en las muestras vegetales.

Procedimiento:

PREPARACIÓN DE LAS MUESTRAS (Maceración del material vegetal)

$\checkmark \quad$ Colocar el material vegetal: de recepción y después de la moliendatamizado en los envases respectivos.

$\checkmark \quad$ Añadir el etanol (Alcohol Potable 96\%) en cada muestra de tal forma que éste cubra completamente el material vegetal e inmediatamente colocar los corchos en los envases.

$\checkmark \quad$ Dejar en maceración, en un lugar cubierto para evitar el contacto con los rayos solares y mantener una temperatura constante durante un tiempo de 15 días.

$\checkmark$ Colocar los extractos etanólicos en los tubos de ensayo respectivamente y sellar los tubos con los tapones.

\section{Espectrometría ir}

$\checkmark \quad$ Medir el pH de cada muestra y comprobar que se encuentre en un rango de 5-9, de lo contrario se debe realizar diluciones del extracto etanólico, esto es muy importante respetar para evitar daños en el equipo.

$\checkmark \quad$ Colocar con la pipeta Pasteur la muestra en el cristal de seleniuro de zinc del ATR previamente limpiándolo con alcohol para cada muestra.

$\checkmark \quad$ Encender el Espectrofotómetro IR y esperar 5 minutos.

$\checkmark \quad$ Ejecutar el programa JASCO para la lectura de espectros.

$\checkmark \quad$ Comparar las longitudes de onda de los espectros de las muestras tanto de la recepción como de la salida de la molienda-tamizado para verificar la conservación de principios en el proceso.

La técnica también se puede trabajar con muestras sólidas obtenidas después de la molienda-tamizado, sin llevar a cabo la maceración de las muestras.

Formulación de la Tisana a partir de las Hojas de Aguacate, Orégano y Fibra de Coco.

Procedimiento:

- Establecimiento experimental de 2 formulaciones en diferentes porcentajes del material vegetal hasta obtener un peso total de $1 \mathrm{~g}$. 
Tabla 1: Formulación de Tisana.

\begin{tabular}{|c|c|c|}
\hline \multirow{2}{*}{ COMIPONDNTE } & F1 & F2 \\
\hline Hojas de orégano & 0,50 & 0,40 \\
\hline Hojas de Aguacate & 0,10 & 0,10 \\
\hline Fibra de Coco & 0,40 & 0,50 \\
\hline Total & 1,00 & 1,00 \\
\hline
\end{tabular}

$\checkmark \quad$ Elaboración de una encuesta de 5 preguntas: 2 de ellas utilizan escalas sensoriales como: nada intenso, poco intenso, normal, intenso y muy intenso; para evaluar color y aroma, las 2 siguientes valoran el sabor y la aceptabilidad, utilizando los siguientes criterios: gusta mucho, gusta poco, ni gusta ni disgusta, disgusta mucho y disgusta poco. La última pregunta considera las alternativas de endulzantes para el consumo de la tisana.

$\checkmark \quad$ Degustación y desarrollo de la encuesta por una muestra de 41 personas mayores de edad, de género masculino y femenino de la Facultad de Ciencias de la ESPOCH.

$\checkmark \quad$ Tabulación de datos y desarrollo del Test de Ordenamiento para la selección de la formulación (Utilización del Programa IBM SPSS Statistics).

\section{Análisis y Discusión de Resultados}

\section{Resultados de la Etapa de Desinfección.}

Los resultados obtenidos en la Tabla 2, indican los valores de $\mathrm{pH}$ de las muestras vegetales en la etapa de desinfección, que tienden a la neutralidad, los cuales según Gennaro plantea que son el indicador de la inexistencia de la carga microbiana y enzimática, la misma se pudo eliminar empleando el dióxido de cloro que tiene las propiedades de destruir enterorvirus, E. coli y amebas y es efectivo contra los quistes de Cryptosporidium, según la información referente al compuesto en mención, expuesta por Deininger, et al (2004). Además al no presentarse como resultado un valor alcalino se revela la desaparición completa del Dióxido de cloro $\left(\mathrm{ClO}_{2}\right)$ cuyo valor de $\mathrm{pH}$ es 14 .

Tabla 2: Valores de $\mathrm{pH}$ de las muestras vegetales en la etapa desinfección.

\begin{tabular}{|c|c|}
\hline Hoja de Orégano & 6,95 \\
\hline Hoja de Aguacate & 7,5 \\
\hline Fibra de Coco & 7,03 \\
\hline
\end{tabular}

\section{Resultados de la Etapa de Deshidratación /Molienda-Tamizado}

Los resultados obtenidos en la Tabla 2, reflejan las características adecuadas a las que deben tratarse los materiales vegetales (hojas de orégano, aguacate y fibra de coco) en el proceso productivo de las tisanas, para obtener un producto de calidad, aumentar su tiempo de vida útil y facilitar su elaboración. Esto se afirma debido a que sus valores coinciden con los 
parámetros manejados por industrias nacionales como: JAMBI KIWA ubicada en la ciudad de Riobamba, ESPECIERÍAS ILE C.A ubicada en la ciudad de Loja y PROCONSUMO C.A. ubicado en la ciudad de Tabacundo, para sus productos y Cholota (2011) con trabajos investigativos de plantas medicinales como: orégano, llantén y menta. Según Sharapin N., la temperatura recomendada para la operación de secado oscila entre los 30 y $60{ }^{\circ} \mathrm{C}$, la cual permite interrumpir procesos de degradación causados por enzimas e impide el desarrollo de microorganismos, en esta etapa puede presentarse pérdida de sustancias volátiles como aceites esenciales, así como el riesgo de degradación de algunas sustancias termolábiles, por ello para garantizar la conservación de todos los principios activos de las plantas la temperatura óptima de secado es de $\quad 30^{\circ} \mathrm{C}$. Según Molina (2014), el tiempo óptimo de secado para plantas aromáticas no debe ser excesivo, ya que la planta puede reducirse a polvo perdiendo sus sustancias activas, en cambio un tiempo escaso, puede provocar descomposición de la materia por la humedad que aún contiene; por tanto un tiempo que oscila entre las 5-8 h de secado, dependiendo de la superficie del material vegetal expuesta al calor, permite obtener una humedad óptima, la cual no debe exceder el $7 \%$ (bh), para facilitar la molienda y sus etapas posteriores.

Tabla 3: Características del Material Vegetal.

\begin{tabular}{|c|c|c|c|c|c|c|}
\hline \multicolumn{2}{|c|}{$x_{b h}(\%)$} & \multicolumn{3}{|c|}{$\overline{\boldsymbol{d}}_{W}(\boldsymbol{m})$} & \multirow{2}{*}{$\begin{array}{c}\text { t (h) } \\
\text { Óptimo } \\
\text { Secado }\end{array}$} & \multirow{2}{*}{$\begin{array}{c}\mathbf{T} \\
\left({ }^{\circ} \mathrm{C}\right)\end{array}$} \\
\hline Inicial & Final & Óptima & Alimentación & Descarga & & \\
\hline \multicolumn{7}{|c|}{ MUESTRA: HOJAS DE ORÉGANO } \\
\hline 68 & 2,8 & 7 & $2,621 \times 10^{-3}$ & $4,123 \times 10^{-4}$ & 7,66 & 30 \\
\hline \multicolumn{7}{|c|}{ MUESTRA: HOJAS DE AGUACATE } \\
\hline 58,4 & 0,73 & 7 & $2,633 \times 10^{-3}$ & $5,887 \times 10^{-4}$ & 8,87 & 30 \\
\hline \multicolumn{7}{|c|}{ MUESTRA: FIBRA DE COCO } \\
\hline $\mathbf{7 8 , 4}$ & 0,88 & 5 & $2,620 \times 10^{-3}$ & $6,458 \times 10^{-4}$ & 6,40 & 30 \\
\hline
\end{tabular}

\section{Resultados de la Granulometría óptima para la tisana}

Las partículas de los elementos vegetales (hojas de orégano, aguacate y fibra de coco) después de la etapa de molienda-tamizado, que atraviesan un tamiz de número de malla $20(850 \mu \mathrm{m}), 40(425 \mu \mathrm{m})$ y $50(300 \mu \mathrm{m})$, fueron utilizadas en la elaboración de la tisana y como resultado revelan que al momento de su infusión instantánea en un medio acuso (agua luego de alcanzar su punto de ebullición) no existe la presencia de sedimentos, lo cual indica que los valores de granulometría del conjunto de partículas cumplen con los requerimientos necesarios para la producción y comercialización de tisanas, esto se puede afirmar al comparar con la granulometría de productos como: Bilgramix, Té de la Abuela, Paico Mix, entre otros, ofertados en el mercado por JAMBI KIWA, la cual cuenta con certificación bajo normas de calidad establecidas, Buenas Prácticas de Manufactura (BPM), certificación 
orgánica con la BSC de Alemania, certificación de Comercio Justo Fair Trade, permiso de Impacto Ambiental con el Ministerio del Ambiente y permiso prefitosanitario otorgado por AGROCALIDAD.

\section{Resultados de la Selección de la Formulación para la tisana}

Los porcentajes de cada uno de los elementos vegetales en formulaciones, se establecieron para un valor de masa total de $1 \mathrm{~g}$, valor considerado por las industrias anteriormente mencionadas en el acápite 3.2, para la elaboración de sus tés aromáticos. Cada formulación estable un porcentaje másico alto de contenido de hojas de orégano, ya que según la norma INEN 2392 ( 2013) es una planta muy recomendada para este tipo de productos y además presenta mejores características organolépticas y contenido en principios activos que las hojas de aguacate y la fibra de coco.

Un análisis sensorial de cada formulación expuesta a posibles consumidores permite medir su aceptación y determinar cuál formulación es la adecuada para la tisana, debido a que la aceptabilidad de un producto es la relación de un consumidor a las propiedades físicas y sensoriales del mismo según Vargas (2012).

En referencia a la muestra de 41 personas los resultados que se presentan en las figuras 1 y 2 muestran que: la tisana de formulación 1 (50\% hojas de orégano, $40 \%$ fibra de coco y $10 \%$ hojas de aguacate) establece una aceptabilidad del 58,54\% según el criterio: gusta mucho, en cambio la tisana de formulación 2 ( $40 \%$ hojas de orégano, $50 \%$ fibra de coco y $10 \%$ hojas de aguacate) presenta una aceptabilidad del 39,02\% según criterios: ni gusta ni disgusta y gusta poco y tan solo un 7,32\% según el criterio: gusta mucho. Al ser notable la diferencia de aceptabilidad entre las tisanas de diferente formulación se da preferencia a la primera.

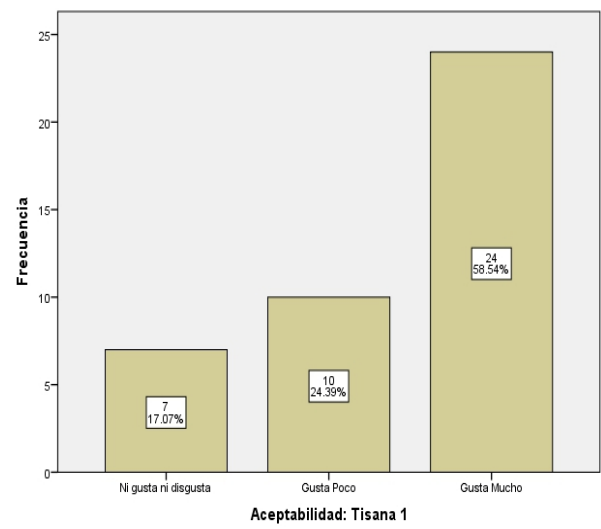

Fig.1: Gráfica Frecuencia vs Aceptabilidad de la Tisana de Formulación 1 


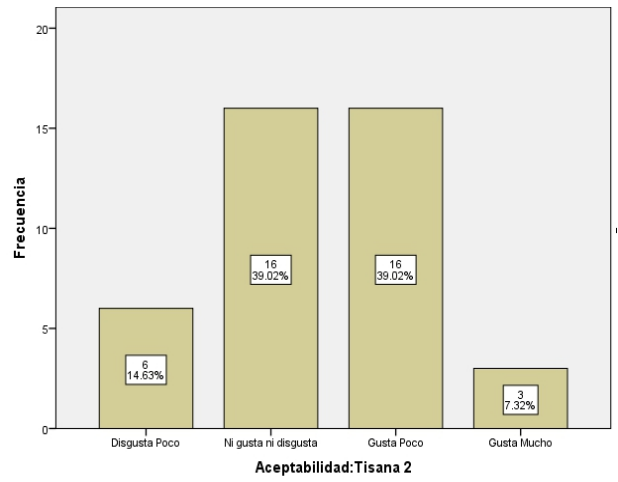

Fig. 2: Gráfica Frecuencia vs Aceptabilidad de la Tisana de Formulación 2

\section{Resultados de la Espectrometría IR.}

Los resultados obtenidos mediante la aplicación de la ESPECTROMETRÍA IR, a las muestras tanto sólidas como en extracto etanólico de las hojas de orégano, aguacate y fibra de coco, establecen espectros infrarrojos, los cuales muestran un cierto número de bandas de absorción asociadas a todas las unidades estructurales de las moléculas orgánicas que constituyen cada una de las muestras, siendo éstas un parámetro de interpretación e identificación de los componentes de dichas muestras debido a la frecuencia característica de sus grupos funcionales.

\section{Espectros de las muestras de las Hojas Orégano.}

De acuerdo al espectro infrarrojo de la muestra de orégano observado en la Figura 3 y conjuntamente con los datos expuestos por Pando E., \& Martín, C., se establece el siguiente análisis cualitativo, mostrándose tensiones de los siguientes grupos funcionales: $\mathrm{O}-\mathrm{H}$ perteneciente a los alcoholes primarios, secundarios, terciarios y fenoles; $\mathrm{C}-\mathrm{H}$ perteneciente a los alcanos como $\mathrm{CH} 3$ y $-\mathrm{CH} 2-$; $\mathrm{C}-\mathrm{O}$ de ésteres y $\mathrm{C}=\mathrm{O}$ de las cetonas, aldehídos y ácidos, que conjuntamente constituyen los compuestos orgánicos como: fenoles (timol y carvacrol), terpenos (cimeno y terpineno) que son los más representativos y demás compuestos como flavonoides y sesquiterpenos.

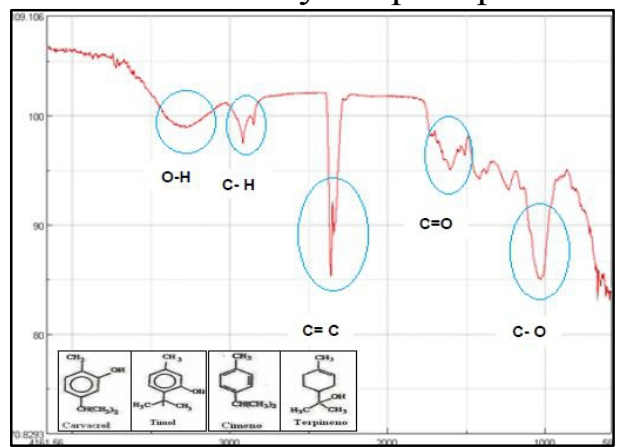

Fig. 3: Espectro IR de muestra sólida del producto de la etapa de molienda. 
Las Figuras 3.a y 3.b, hacen una comparación de las bandas más sobresalientes de los espectros infrarrojos de las muestras de las hojas de orégano en la etapa de recepción y del producto de la etapa de molienda, indicándose que al presentar las mismas frecuencias de sus grupos funcionales se asegura la conservación de todos sus principios activos durante todo el proceso de elaboración de las tisanas.

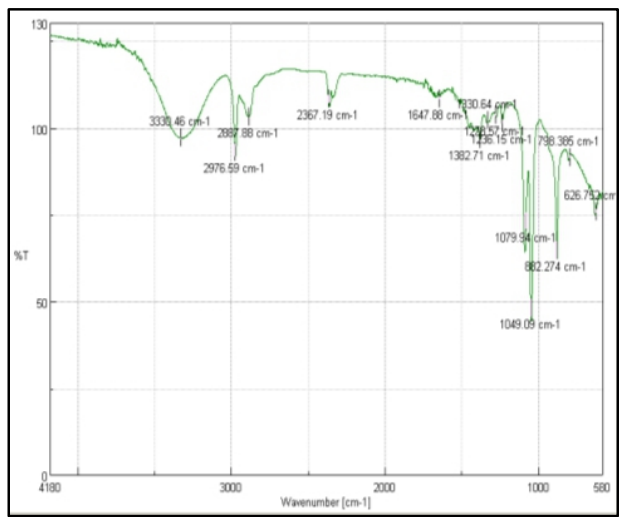

Fig. 3.a: Espectro IR del extracto etanólico de la muestra de la recepción.

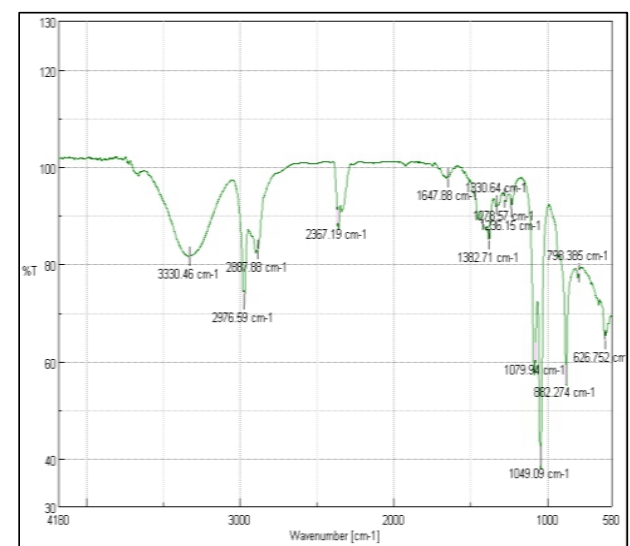

Fig. 3.b: Espectro IR del extracto etanólico de la muestra del producto de la etapa de molienda.

\section{Espectros de las muestras de las Hojas de Aguacate.}

De acuerdo al espectro infrarrojo de la muestra de aguacate observado en la Figura 4 y conjuntamente con los datos expuestos por Pando E., \& Martín, C., se establece el siguiente análisis cualitativo, mostrándose tensiones de los siguientes grupos funcionales: $\mathrm{C}-\mathrm{H}$ perteneciente los alcanos como $\mathrm{CH} 3$ y $-\mathrm{CH} 2-; \mathrm{C}=\mathrm{C}$ perteneciente al doble enlace carbono de los compuestos aromáticos; $\mathrm{C}-\mathrm{O}$ de ésteres y $\mathrm{C} 6 \mathrm{H} 6$ del anillo bencénico, que conjuntamente constituyen los compuestos como: fenilpropanos (estragol), sesquiterpenos (cariofileno), monoterpenos (cimeno y pineno) que son los más representativos. 


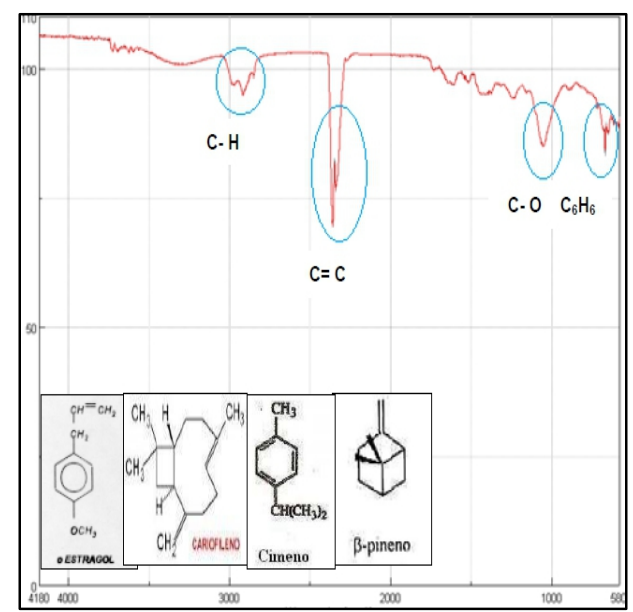

Fig. 4: Espectro IR de la muestra sólida del producto de la etapa de molienda.

Las Figuras 4.a y 4.b, hacen una comparación de las bandas más sobresalientes de los espectros infrarrojos de las muestras de las hojas de aguacate en la etapa de recepción y del producto de la etapa de molienda, indicándose que al presentar las mismas frecuencias de sus grupos funcionales se asegura la conservación de todos sus principios activos durante todo el proceso de elaboración de las tisanas.

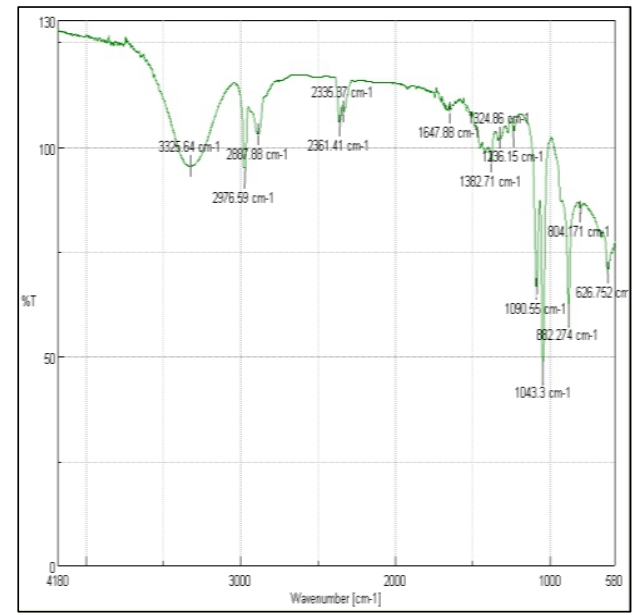

Fig. 4.a: Espectro IR del extracto etanólico de las hojas de aguacate de la recepción. 


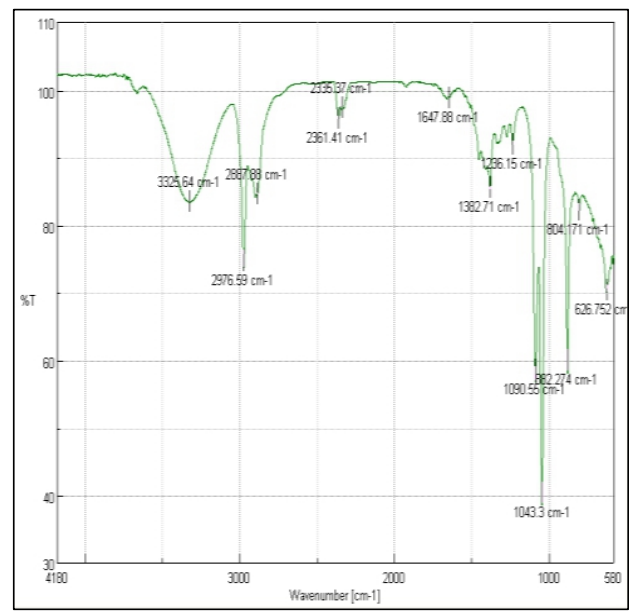

Fig. 4.b: Espectro IR del extracto etanólico de la muestra del producto de la etapa de molienda.

Espectros de las muestras de la Fibra de Coco

De acuerdo al espectro infrarrojo de la muestra de la fibra de coco observado en la Figura 5 y conjuntamente con los datos expuestos por Pando E., \& Martín, C., se establece el siguiente análisis cualitativo, mostrándose tensiones de los siguientes grupos funcionales: $\mathrm{C}-\mathrm{H}$ perteneciente a la tensión de alcanos como $-\mathrm{CH} 3$ y $-\mathrm{CH} 2-; \mathrm{C}=\mathrm{C}$ perteneciente a la tensión de doble enlace carbono de los compuestos aromáticos; $\mathrm{C}-\mathrm{O}$ de ésteres, que conjuntamente constituyen los compuestos como: polisacáridos (lignina, celulosa y pentanosa), que son los más representativos.

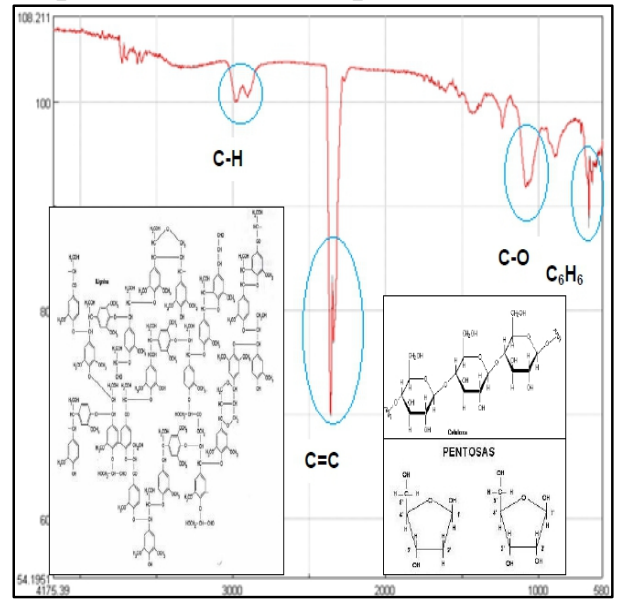

Fig. 5: Espectro IR de la muestra sólida del producto de la etapa de molienda.

Las Figuras 5.a y 5.b, hacen una comparación de las bandas más sobresalientes de los espectros infrarrojos de las muestras de la fibra de coco en la etapa de recepción y del producto de la etapa de molienda, indicándose 
que al presentar las mismas frecuencias de sus grupos funcionales se asegura la conservación de todos sus principios activos durante todo el proceso de elaboración de las tisanas.

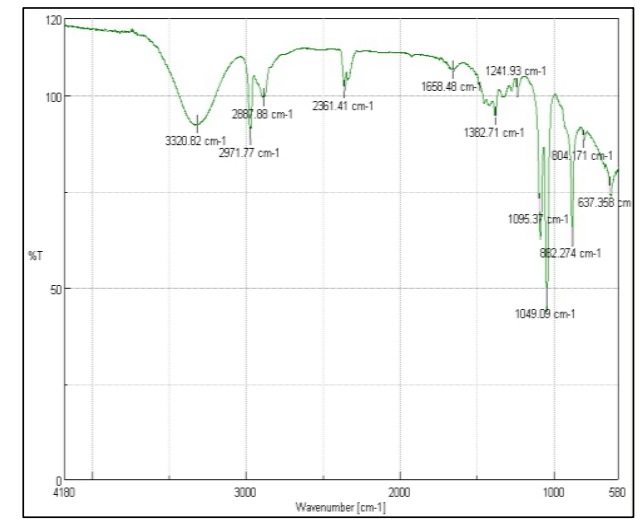

Fig. 5.a: Espectro IR del extracto etanólico muestra de la recepción.

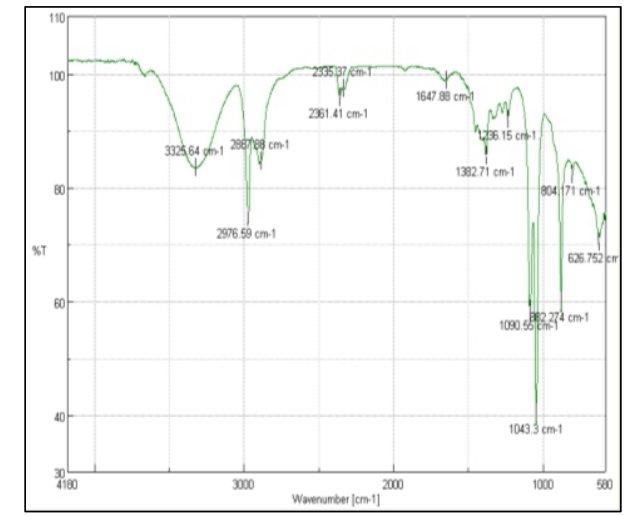

Fig. 5.b: Espectro IR del extracto etanólico de la muestra del producto de la etapa de molienda.

\section{Resultados del Análisis Estadístico Inferencial.}

$\mathrm{Al}$ aplicar una prueba $\mathrm{X}^{2}$ Chi Cuadrada de Pearson con dos grados de libertad y un $95 \%$ de confiabilidad se determinó que existe independencia entre las variables de aceptabilidad de la tisana (formulación 1) y el género del consumidor, debido a que según Hernández R. (2010), indica que a un porcentaje mayor al 5\% de significancia asintótica bilateral se acepta la hipótesis nula y se verifica la independencia entre las variables manejadas. En relación al consumo de la tisana de formulación seleccionada, por estudiantes politécnicos encuestados, se establece que: al $65,10 \%$ gustó mucho siendo las respuestas semejantes tanto en hombres como en mujeres, el 21,6\% gustó poco y el 13,27\% ni gustó ni disgustó; valores que se reflejan en la Figura 5. 
Tabla 4: Prueba $\mathrm{X}^{2}$ Chi-Cuadrada.

\begin{tabular}{|c|c|c|c|}
\hline & Valor & gl & Sig. asintótica (bilateral) \\
\hline Chi-cuadrado de Pearson & $.252^{\mathrm{a}}$ & 2 & .882 \\
\hline Razón de verosimilitudes & .252 & 2 & .882 \\
\hline Asociación lineal por lineal & .215 & 1 & .643 \\
\hline N de casos válidos & 324 & & \\
\hline
\end{tabular}

a. 0 casillas $(0.0 \%)$ tienen una frecuencia esperada inferior a 5. La frecuencia mínima esperada es 21.50 .

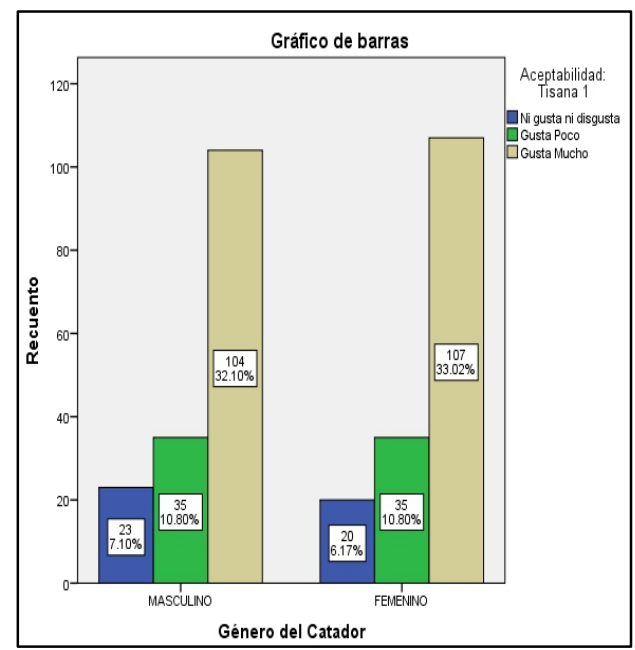

Fig. 6: Resultados de la Aceptabilidad de la tisana 1 según el Género del Consumidor

\section{Conclusiones}

$>\quad$ Los valores de $\mathrm{pH}$ con tendencia a la neutralidad, obtenidos en la etapa de desinfección concluyen la inexistencia de carga microbiana y enzimática, así como la desaparición del $\mathrm{ClO}_{2}$.

$>\quad$ La humedad óptima de los materiales vegetales previo a la moliendatamizado son: $7 \%$ (bh) para las hojas y 5\%(bh) para la fibra.

$>\quad$ El diámetro promedio en masa inicial del material vegetal corresponde a los siguientes valores: $2,621 \times 10^{-3} \mathrm{~m}$ hojas de orégano, $2,633 \times 10^{-3} \mathrm{~m}$ hojas de aguacate y $2,620 \times 10^{-3} \mathrm{~m}$ fibra de coco.

$>\quad$ El diámetro promedio en masa final de las partículas posterior a la molienda-tamizado es: $4,123 \times 10^{-4} \mathrm{~m}$ (hojas de orégano), 5,887 $\times 10^{-4} \mathrm{~m}$ (hojas de aguacate) y $6,458 \times 10^{-4} \mathrm{~m}$ (fibra de coco).

$>\quad$ La igualdad de longitud de onda de los picos más sobresalientes de los espectros de las muestras de recepción y molienda-tamizado concluyen la conservación de principios activos de los elementos vegetales.

$>$ La composición por gramo de tisana es: $50 \%$ hojas de orégano, $40 \%$ fibra de coco y $10 \%$ hojas de aguacate. 
$>\quad$ La degustación del producto concluye las siguientes características organolépticas: color anaranjado poco intenso, olor característico de elementos vegetales y sabor equilibrado de los componentes.

$>\quad$ El producto presentó un porcentaje de aceptabilidad del $65,12 \%$, entre los géneros: masculino y femenino.

\section{Recomendaciones}

Una vez establecido el producto final y verificando su aceptabilidad se recomienda realizar el estudio acerca de la efectividad de la tisana para aliviar malestares digestivos y tratar problemas menstruales.

\section{References:}

1. Berdonces, J. L. (2010). Gran Diccionario de las Plantas Medicinales: Descripción Aplicaciones. España: Océano Ambar.

2. Cajas, D. B. (2011). Diseño de un Triturador Pulverizador de Estopa de Coco para la producción de Sustrato Granulado. Tesis de Pregrado, Escuela Politécnica Nacional, Facultad de Ingeniería Mecánica, Quito.

3. Cholota, J. (2011). Obtención de Té Medicinal Nutracéutico a partir de plantas ancestrales Menta (Mentha arvensis) Manzanilla (Matricaria chamomilla) Llantén (Plantaginaceae) Malva (Malváceas o malvaceae). Universidad Técnica de Ambato, Facultad de Ciencia e Ingeniería en Alimentos, Ambato.

4. Deininger, R. A., Ancheta, A., \& Ziegler, A. (s.f.). bvsde.ops-oms. Recuperado el 31 de 05 de 2015, de Dióxido de Cloro: http://www.bvsde.opsoms.org/bvsacg/e/fulltext/simposio/ponen11.pdf

5. Hernández, R. F. (2010). Metodología de la Investigación. En R. F. Hernández, Metodología de la Investigación (5 ed., págs. 327-331). Mc Graw Hill.

6. INEN. (2013). Hierbas Aromáticas. Requisitos. Quito.

7. Marcillo, E., \& Naranjo, E. (2012). Diseño de la Línea de Producción de una Bebida de Hierbas Denomidana Horchata. Tesis de Pregrado, Escuela Politécnica del Litoral, Facultad de Ingeniería en Mecánica y Ciencia de la Producción, Guayaquil.

8. Molina Sánchez, J. I. (2014). Mejoramiento de la Producción de Helados de Uvilla (Physalis peruviana), y Desarrollo de Néctar, Bebida no Carbonatada, Té de Uvilla y Deshidratación Osmótica de la Fruta. Tesis de Pregrado, Universidad Técnica de Ambato, Facultad de Ciencia e Ingeniería en Alimentos, Ambato.

9. Ocon, J., \& Tojo, G. (1986). Problemas de Ingeniería Química. Aguilar. 
10. Pamplona, J. (2006). Enciclopedia de las Plantas Medicinales. Argentina: Safeliz.

11. Pando, E., \& Martín, C. (1979). Problemas de determinación estructural orgánica por espectrometría IR. Salamanca: Universidad de Sevilla.

12. Sánchez, E. (2013). Evaluación de Biofertilizante en el Cultivo de Orégano (Origanum vukkgare L.) en la Granja Experimental Querochaca. Trabajo de Investigación, Universidad Técnica de Ambato, Facultad Cienias Agropecuarias, Ambato.

13. Sánchez, M. (2009). Estudio invetigativo del orégano: producción, cultivo, análisis de sus propiedades y su aplicación en la gastronomía ecuatoriana. Tesis de Pregrado, Universidad Tecnológica Equinoccial, Facultad de Turismo y Preservación Ambiental, Hotelería y Gastronomía, Quito.

14. Tonguino, I. (2011). Determinación de las condiciones óptimas para la deshidratación de dosplantas aromáticas; Menta (Mentha piperrita L.) y Orégano (Origamun vulgare L.). Tesis de Pregrado, Univesidad Técnica del Norte, Facultad de Ingeniería en Ciencias Agropecuarias y Ambientales, Ibarra.

15. Torrenegra, M. (2014). Evaluación de la Atividad Antioxidante del aceite esencial foliar extraído de especies de orégano (Orégano vulgare), orégano "borde blanco" (Orégano vulgare ssp.) y oreganito (Lippia) cultivada en la zona norte del Departamento de Bolivar. Tesis de Maestría en Ciencias y Tecnología de Alimentos, Departamento d Ingeniería Agrícloa y de Alimentos, Bogotá.

16. Valdez, J. (2011). Determinación del contenido, variación fenológica y efecto citotóxico de persina de aguacate criollo mexicano (Persea amiricana Mill. var.drymifolia).

17. Vargas Corrales, V. (2012). Elaboración de té aromático a base de plantas cedrón (aloysiacitrodora) y toronjil (mellisaofficinalis) procesado con stevia (steviarebaudiana bertoni) endulzante natural, utilizando el método de deshidratación. Universidad Técnica de Cotopaxi, Ingeniería Agroindustrial. Latacunga: UTC. 